\title{
A new cellular type in invertebrates: first evidence of telocytes in leech Hirudo medicinalis
}

\author{
Laura Pulze', Miriam Capri ${ }^{2}$, Annalisa Grimaldi', Stefano Salvioli' ${ }^{2}$, Gianluca Tettamanti', Magda de Eguileor ${ }^{1 *}$ \\ University of Insubria, Department of Biotechnology and Life Sciences, 21100 Varese, Italy \\ ${ }^{2}$ University of Bologna, Department of Experimental, Diagnostic and Specialty Medicine (DIMES), 40126 Bologna, Italy
}

Article Info

\section{Article Notes}

Received: December 21, 2017

Accepted: January 16, 2018

\section{*Correspondence:}

Dr. Magda de Eguileor, Department of Biotechnology and Life Sciences, University of Insubria, Via J. H. Dunant 3,

21100 Varese, Italy;

Telephone: +390332 421310;

FAX: +390332 421300,

E-mail: magda.deeguileor@uninsubria.it

C 2018 de Eguileor M. This article is distributed under the terms of the Creative Commons Attribution 4.0 International License.
The leeches (Hirudo medicinalis) have proven to be a good model for deciphering basic biological processes for two main reasons: first of all, they have a reduced dimension and, despite a relative anatomical simplicity, share with vertebrates the complexity of immunological mechanisms and wound-healing processes; secondly, in animal kingdom there is a remarkable evolutionary conservation of biological responses, cell types, cellular mechanisms, and molecules. One of the most phylogenetically conserved system, from lower invertebrates to man, is the innate immune system that, strictly interacting with neuro-endocrine one, guarantees a powerful protection to organisms ${ }^{1-3}$.

A wide range of invertebrate immunocytes share with vertebrate phagocytic macrophages and cytotoxic Natural killer (NK) cells, migration ability, chemotaxis, phagocytosis, and cytotoxicity in response to stress, wound-healing or transplantation ${ }^{4,5}$. Once stimulated, invertebrate immunocytes synthesize molecules typical of the immune and neuroendocrine systems, together with a large amount of peptides ${ }^{3-9}$.

In our paper, here commented ${ }^{10}$ we have provided evidences about the presence in the leech of telocytes (TC), a new type of cell involved in surveillance and protection that has just been recently described as ubiquitous in vertebrates, including humans ${ }^{11-14}$.

As in vertebrate species, leech TCs are stromal cells (distinct from macrophages, NK cells, fibroblasts) spread in various tissues and strategically localized among resident cells, nearby the capillaries and the nerve endings. These cells, organized in an extensive threedimensional network, show direct interactions with adjacent cells via gap junctions, and contacts with the surrounding basal lamina via hemidesmosomes and with collagen of connective tissue via proteoglycans ${ }^{10}$.

The networking among TCs themselves and with different cellular types is essentially due to their morphological characteristics. Leech telocytes show a tiny spindle-shaped cell body from which the telopods, very long convoluted cytoplasmic processes, originate. The interaction among these and the other cells is obtained in two ways: physically, by direct cell-cell contacts and, chemically, via the release of microvesicles and exosomes, which can transport a variety of soluble factors involved in the regulation of different physiological 
processes ${ }^{10}$.

Immunophenotype of TCs is quite complex ${ }^{11-13}$. We have demonstrated ${ }^{10}$ that leech TCs display a doublepositive immunostaining with CD34/vimentin, c-kit/ vimentin, c-kit/CD34 and c-kit/Oct-4, which are stem cell markers expressed simultaneously with the hallmarks of TCs. Moreover leech TCs, as various types of immunocytes belonging to different invertebrate taxa, express markers of the immune-surveillance such as Toll-like receptors (TLRs) 4 and 5, allograft inflammatory factor-1 (Aif-1 also known as IBA-1) involved in inflammatory responses, adrenocorticotropic hormone (ACTH) implicated in the immune and neuroendocrine responses, and endogenous pro-inflammatory cytokines, such as IL-18.

Leech-TCs, once activated in response to chemical or physical stimuli, are able to change their morphology and behaviour, acquiring migratory phenotype, and overexpress the previously mentioned markers. During angiogenesis phase characterizing graft rejection [15-17] and wound healing [10], H. medicinalis telocytes originating from circulating precursor cells, rapidly move away from the capillary lumen toward the injured area where they accumulate and participate in repair/regenerative processes $^{10}$.

Taken together the data presented in the commented paper $^{10}$, integrated with current literature on vertebrates $^{11-14}$, not only provide evidence for the existence of a 3D TC network in the examined invertebrate model, analogous to that of vertebrates, but also reinforce the concept that this evolutionarily conserved system, formed by resident cells, forms an extended network aimed at the recognition of danger signals from the external or endogenous environment. Once activated, the first response of $\mathrm{H}$. medicinalis TCs results in alert messages circulating in all directions and involving any body districts

The TC immuno-surveillance system, obtained by direct cell-cell contacts and soluble mediators released in loco, controls and drives the activity of different cell types in different body regions. The 3D network of TCs, equipped to function as an immune-neuro-endocrine system, is able to respond promptly and faster than other types of immunocytes due to their residentiality. Macrophages and NK cells are activated and become operative, showing a migratory and proliferative phenotypes, in response to soluble signals derived by the cells directly involved in lesioned or injured sites and slowly spreading through the matrix.

Leech TCs, that share the same mesodermal lineage with various types of invertebrate/vertebrate immunocytes, are part of innate immunity and play an important role in monitoring the self/non-self, but they could also be involved in archiving data pertaining to external or internal threats. We have already hypothesized the existence of a form of memory in allo- and xeno-transplantation in leeches because the responses were always faster and stronger in second-set grafting experiments than in the first-set ones ${ }^{17}$. For these reasons it is tempting to speculate that 3D-TC network, with the capability to integrate many different functions, might also play a role in innate immune memory (also indicated as "trained immunity"). Trained immunity is defined as the capacity of innate immune system to respond more effectively not only to previously encountered insults, but also to new, unrelated ones ${ }^{18}$. It involves cells devoid of specific antigen receptors that, until some years ago, were considered unable to mount memory responses (myeloid cells, NK) cells, innate lymphoid cells (ILCs). At variance with classical immunological memory, trained immunity makes use of germline-encoded pattern recognition receptors such as Toll-Like Receptors (TLRs) and epigenetic mechanisms of cell reprogramming ${ }^{19}$.

A growing number of studies suggest that TCs are involved in a variety of processes mediated by homo- and heteromechanical and electrochemical communications ${ }^{11}$. Indeed, a very provocative idea arises from the expanded comparison among leech TCs, leech microglia ${ }^{20,21}$ and vertebrate microglia ${ }^{22-25}$. All these cells present a number of similarities in morphology, cellular functions, cell surface antigens, histochemical properties, mesodermal origin, capability to migrate, proliferate and differentiate in response to injury (see table 1). Interestingly, microglia (from invertebrates ${ }^{20,21,26}$ and vertebrates ${ }^{22-25}$ ) and TCs can be considered as the ancient, conserved interface between immune and neuroendocrine systems as they share similar mediators and receptors, from lower invertebrates up to human. Furthermore, as TCs have receptors, such as TLR-4,

Table 1: Comparison between morphology, dimensions, cell surface markers, specific molecules useful for the identification and characterization of telocytes and microglia.

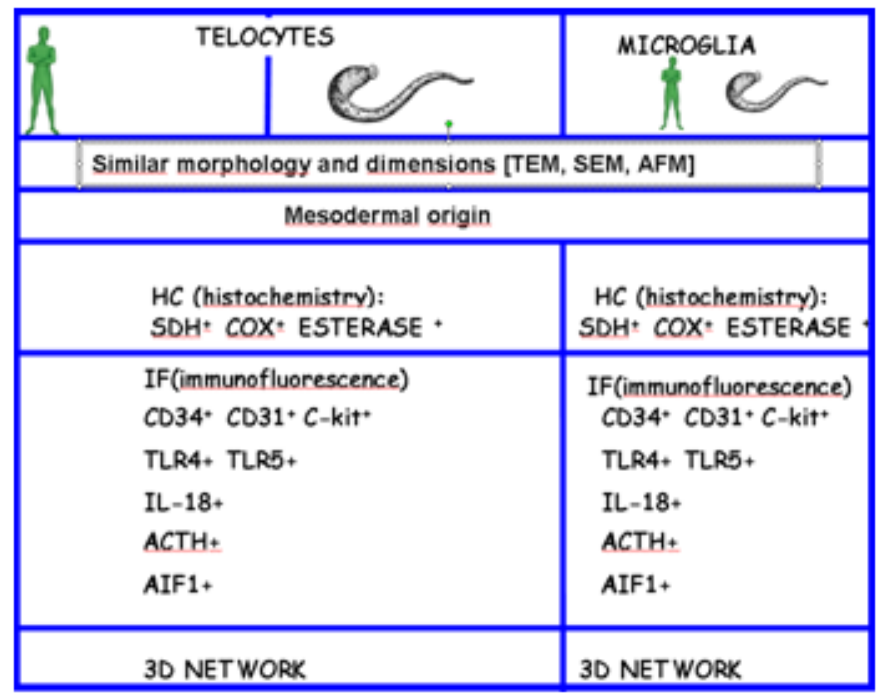


and -5 potentially relevant to trigger signalling that makes them primed and trained for a faster response to a second antigen exposure, it is possible that TCs as a primordial network of defence share some functional features of trained immunity with other more popular innate immune cells such as NK cells and macrophages ${ }^{27}$. In this perspective, TCs could be considered as non-terminally differentiated cells forming a sort of primordial network with features of immature neuro/immune-cells.

Thus, it can be hypothesised that TCs and microglial cells could represent the same type of cells resident in different districts, able to detect the first signs of invasion or tissue damage, and to provide a rapid and quick first-line response to these threats. Furthermore, considering the apparently universal diffusion of the 3D telocyte network, it is likely that it serves as a primordial, fundamental defence and cell-to-cell communication system, that could be directly or indirectly involved in the pathogenesis of disorders characterized by inflammatory profile, such as several heart, lung and intestinal diseases ${ }^{28-31}$. If this hypothesis is confirmed, the biological (and maybe also clinical) relevance of our speculations might be to be taken into serious consideration and a new chapter of the local/ organ-related immunity will be written.

\section{Acknowledgements}

This study was technically supported by Centro Grandi Attrezzature (CGA) core facilities of University of Insubria.

The authors' research was funded by CARIPLO (Fondazione Cassa di Risparmio delle Province Lombarde) - 2016-0835 Ricerca malattie invecchiamento 2016: FRAMYEVO.

\section{References}

1. ThyagaRajan, Priyanka HP. "Bidirectional communication between the neuroendocrine system and the immune system: relevance to health and diseases". Annals of Neurosciences. 2012; vol.19: pp.4046.

2. Ottaviani E, Malagoli D, Franceschi C. "Common evolutionary origin of the immune and neuroendocrine systems: from morphological and functional evidence to in silico approaches". Trends Immunol. 2007; vol. 28: pp.497-502.

3. Ottaviani E, Franceschi C. "The invertebrate phagocytic immunocyte:clues to a common evolution of immune and neuroendocrine systems". Immunol Today. 1997; vol.18: pp.169-174.

4. Tascedda F, Ottaviani E. "Biologically active peptides in molluscs". Inv Surv J. 2016; vol.13: pp.186-190.

5. de Eguileor M, Tettamanti G, Grimaldi A, et al. "Leeches: Immune response, angiogenesis and biomedical applications". Curr Pharm Des. 2003; vol.9: pp. 133-149.

6. Vaure C, Liu YA. “Comparative review of Toll-like receptor 4 expression and functionality in different animal species". Front Immunol. 2014; vol. 5: pp. 316-322.

7. Coscia MR, Giacomelli S, Oreste U. “Toll-like receptors: an overview from invertebrates to vertebrates". Invert Surv J. 2011; vol. 8: pp. 210226.
8. Schorn T, Drago F, Tettamanti G, et al. "Homolog of allograft inflammatory factor- 1 induces macrophage migration during innate immune response in leech". Cell Tissue Res. 2015; vol. 359 (3): pp. 853-864. doi: 10.1007/s00441-014-2058-7,

9. Bossù $\mathrm{P}$, Ciaramella $\mathrm{A}$, Salani $\mathrm{F}$, et al. "Interleukin-18, from neuroinflammation to Alzheimer's disease". Curr Pharm Des. 2010; vol. 16: pp. 4213-4224. ISSN: 1824-307X

10. Pulze L, Baranzini N, Girardello R et al. "A new cellular type in invertebrates: first evidence of telocytes in leech Hirudo medicinalis". Sci Rep. vol. 7: pp. 13580-13592.

11. Cretoiu D, Radu BM, Banciu A, et al. "Telocytes heterogeneity: From cellular morphology to functional evidence," Semin. Cell Dev Biol. 2017; vol. 64: pp. 26-39.

12. Popescu LM, Manole E, Serboiu CS, et al. " Identification of telocytes in skeletal muscle interstitium: implication for muscle regeneration". J Cell Mol Med. 2011; vol. 15: pp.1379-1392.

13. Cretoiu SM, Popescu LM. “Telocytes revisited". Biomol Concepts. 2014; vol. 5: pp.353-369.

14. Vannucchi MG, Bani D, MS. “Telocytes Contribute as Cell Progenitors and Differentiation Inductors in Tissue Regeneration". Curr Stem Cell Res Ther. 2016; vol.11: pp.383-389.

15. Grimaldi A. "Origin and fate of hematopoietic stem precursor cells in the leech Hirudo medicinalis". Invert Surv J. 2016; vol.13: pp.257-268.

16. Tettamanti G, Grimaldi A, Valvassori R, et al. "Vascular endothelial growth factor is involved in neoangiogenesis in Hirudo medicinalis (Annelida, Hirudinea)". Cytokine. 2003; vol. 22: pp.168-179.

17. Tettamanti G, Grimaldi A, Ferrarese R, et al. "Leech responses to tissue transplantation". Tissue and Cell. 2003; vol. 35: pp. 199-212.

18. Netea MG, Quintin J, van der Meer JW. "Trained immunity: a memory for innate host defense". Cell Host Microbe. 2011; vol. 9: pp. 355.

19. Netea MG, Joosten LA, Latz E, et al. "Trained immunity: A program of innate immune memory in health and disease". Science. 2016; vol. 352: pp. 1-11.

20. Le Marrec-Croq F, Drago F, Vizioli J, et al. "The leech nervous system: A valuable model to study the microglia involvement in regenerative processes". Clin Dev Immunol. 2013; vol. 27: pp.4019.

21. Drago F, Sautière PE, Le Marrec-Croq F, et al. "Microglia of medicinal leech (Hirudo medicinalis) express a specific activation marker homologous to vertebrate ionized calcium-binding adapter molecule 1 (Iba1/alias Aif-1)”. Dev Neurobiol. 2014; vol. 74: pp. 987-1001.

22. Olson JK, Miller SD. "Microglia initiate central nervous system innate and adaptive immune responses through multiple TLRs". J Immunol. 2004; vol. 173: pp. 3916-3924.

23. Raivich G, Banati R. "Brain microglia and blood-derived macrophages: molecular profiles and functional roles in multiple sclerosis and animal models of autoimmune demyelinating disease". Brain Res Rev. 2004; vol. 46: pp. 261-281.

24. Lehnardt S. "Innate immunity and neuroinflammation in the CNS: the role of microglia in Toll-like receptor-mediated neuronal injury". 2010; vol.58: pp. 253-263.

25. Ginhoux F, Lim S, Hoeffel G, et al. "Origin and differentiation of microglia” Front. Cell Neurosci., vol. 7, pp. 45-52, 2013.

26. D.Sonetti, et al., "Microglia in invertebrate ganglia". PNAS. 1994; vol.91: pp. 9180-9184.

27. C. Franceschi, S. Salvioli, P. Garagnani, M. de Eguileor, D. Monti and M. Capri. "Immunobiography and the Heterogeneity of Immune Responses in the Elderly: A Focus on Inflammaging and Trained Immunity". Front Immunol. 2017; vol.8: pp, 982-1005.

28. Manole CG, Cismasiu V, Gherghiceanu M, et al. "Experimental 
acute myocardial infarction: telocytes involvement in neoangiogenesis". J Cell Mol Med. 2011; vol.15: pp.2284-2296.

29. Milia F, Ruffo M, Manetti M, et al. "Telocytes in Crohn's disease”. J
Cell Mol Med. 2013; vol.17: pp.1525-1536.

30. Zhao B . "Cardiac telocytes were decreased during myocardial infarction and their therapeutic effects for ischemic heart in rat". J Cell Mol Med. 2013; vol.17: pp.123-133.

31. Song D, Cretoiu D, Cretoiu SM, et al. "Telocytes and lung disease", Histol Histopathol. 2016; vol. 31: pp.1303-1314. 